\title{
Occurrence and detection of AmpC $\beta$-lactamases among some clinical isolates of Enterobacteriaceae obtained from Mansoura University Hospitals, Egypt
}

\author{
Barwa R.*, Abdelmegeed E. and Abd El Galil K. \\ Department of Microbiology, Faculty of Pharmacy, Mansoura University, Mansoura, Egypt.
}

Accepted 9 June, 2012

\begin{abstract}
The increasing incidence of $\beta$-lactam resistance due to AmpC $\beta$-lactamases in Egypt necessitated this study which aimed to evaluate four different phenotypic methods for detection of AmpC $\beta$-lactamases among some clinical isolates of Enterobacteriaceae and compare these results with those obtained using polymerase chain reaction. The distribution of five AmpC $\beta$-lactamases genes (AmpC, CIT-M, Fox1, $A C C-1, A C T-1)$ were determined among the clinical isolates. Among 180 clinical isolates of Enterobacteriaceae, only 57 isolates were AmpC producers by phenotypic methods and 108 were AmpC producers by polymerase chain reaction. Phenotypic methods adapted in this study gave variable results with the most discriminatory results given by direct inoculation of both the enzyme extract and the bacterial culture in the wells. Of these, the best results were given by enzyme inoculation methods where $\mathbf{4 3}$ isolates exhibited positive result by this method. The distribution of AmpC $\beta$-lactamases gene among the clinical isolates showed that $A m p C$ gene predominated in Escherichia coli. Fox gene was predominantly present in Enterobacter cloacae and E. coli isolates. ACT-1 predominated in E. cloacae. In contrast, enzymes from $C I T-M$ and $A C C-1$ group were rarely present in Enterobacteriaceae.
\end{abstract}

Key words: Betalactam resistance, Enterobacteriaceae, AmpC betalactamases, polymerase chain reaction.

\section{INTRODUCTION}

AmpC $\beta$-lactamases are clinically important enzymes because they may be associated with the resistance to a wide variety of $\beta$-lactam drugs except carbapenems and cefepime (Odeh et al., 2002). AmpC enzymes class $\beta$ lactamases hydrolyze penicillins, cephalosporoins and cephamycins such as cefoxitin or cefotetan. They can also hydrolyze oxyimino cephalosporins such as ceftazidime, cefotaxime and ceftriaxone and monobactams such as aztreonam but the hydrolysis rates for cefepime, cefpirome and carbapenems are very low (Coudron et al., 2000). They differ from extended-spectrum $\beta$-lactamases (ESBLs) by their ability to hydrolyze cephamycins and they are not affected by available $\beta$-lactamase inhibitors (Manchanda and Singh, 2003). Genes of AmpC $\beta$-lactamases were encoded on the chromosomes of several

\footnotetext{
${ }^{*}$ Corresponding author. E-mail: rasha2000@gmail.com. Tel: +20502343504 . Fax +20502247496
}

members of the family Enterobacteriaceae (Barlow and Hall, 2002). The bacteria lack or poorly express a chromosomal bla $A m p C$ gene that may contain transmissible plasmids that carry acquired genes for AmpC enzymes (Jacoby, 2009). Plasmids with these genes can spread among members of the family Enterobacteriaceae and have been documented in many countries (Coudron, 2003). Plasmids carrying genes for AmpC $\beta$-lactamases often carry multiple resistances including genes for resistance to aminoglycosides, quinolones, chloramphenicol, sulfonamide, tetracycline, and trimethoprim as well as genes for other $\beta$ - lactamases such as CTX-M-3 (Chen et al. 2007).

The ability to detect AmpC enzymes is important to improve the clinical management of infections, but at present, there are no standardized phenotypic screening methods that are readily available for detection of these enzymes. Increased resistance to cefoxitin in the Enterobacteriaceae may be an indicator of AmpC activity, but cefoxitin resistance may also be mediated through altera- 
tions of outer membrane permeability (Hernandez-Alles et al., 2000). Various workers used double disc-synergy and three dimensional extract test for detection of AmpC harboring Gram-negative organisms. Since it was found that the disc diffusion test was non specific and three dimensional extract test is a labour-intensive procedure, there is always a search for newer methods and the aim is to make existing methods more user-friendly to incorporate them in routine diagnostic laboratories (Manchanda and Singh, 2003).

The objective of the present study is to establish a user friendly and effective protocol for the detection of AmpC enzymes. This was attained through a comparative study between the reported phenotypic screening methods and genotypic detection methods using PCR.

\section{MATERIALS AND METHODS}

\section{Clinical isolates}

A total of 180 clinical isolates of Enterobacteriaceae were collected from different patients distributed among university hospitals in Mansoura, Dakahlia governorate, Egypt. The isolates were collected from urine and sputum samples. These isolates were identified as Escherichia coli (87 isolates), Klebsiella pneumoniae (61 isolates) and Enterobacter cloacae (32 isolates) using standard biotyping methods (Crichton, 1996). All isolates were tested for AmpC $\beta$-lactamase production using phenotypic and genotypic methods.

\section{Phenotypic methods for detection of AmpC $\beta$-lactamase activity}

AmpC enzyme production was estimated by modified threedimensional test (M3D) with several modifications in previous works (Coudron et al., 2000; Manchanda and Singh, 2003). Cefoxitin (30 $\mu \mathrm{g})$ discs were used for AmpC enzyme detection. The methods were divided into two groups:

\section{Methods directly using the bacterial isolates}

Lawn cultures of sensitive E. coli were prepared on Mueller-Hinton agar and the plates were incubated for $15 \mathrm{~min}$ at $37^{\circ} \mathrm{C}$, for drying. Then $30 \mu \mathrm{g}$ cefoxitin disc was placed on the surface of the inoculated $\mathrm{MH}$ agar plate using a sterile forceps. The adopted methods were:

Method 1: Linear slits $(3 \mathrm{~cm})$ were cut using a sterile surgical blade $3 \mathrm{~mm}$ away from the edge cefoxitin disc. Small circular wells were made on the slits at $5 \mathrm{~mm}$ distance, inside the outer edge of the slit, by stabbing with a sterile pasture pipette on the agar surface. The method was modified by making the wells within, outside and approximately at the margin of the zone of inhibition with and without linear slit. The well was loaded approximately with 30 to 40 $\mu \mathrm{l}$ of 0.5 Mcfarland bacterial suspension prepared from an overnight broth suspension of the tested isolates in $10 \mu \mathrm{l}$ increments until the well was filled to the top. The plate was kept upright for 5 to $10 \mathrm{~min}$ until the solution dried, and was then incubated at $37^{\circ} \mathrm{C}$ overnight (Manchanda and Singh, 2003).
Method 2: A heavy inoculum of the tested isolate was streaked over the agar surface in a linear fashion, beginning $5 \mathrm{~mm}$ from cefoxitin disc and moving outwards. The plate was then incubated at $37^{\circ} \mathrm{C}$ overnight (Shahid et al., 2004).

Method 3: Five to six colonies of the tested isolate were spot inoculated at a distance of 9 to $10 \mathrm{~mm}$ from the edge of cefoxitin disc approximately at the zone of inhibition. The plate was then incubated at $37^{\circ} \mathrm{C}$ overnight (Shahid et al., 2004).

\section{Methods using the enzyme extract (three dimensional extract method)}

Lawn cultures of $E$. coli were prepared as described previously and cefoxitin $(30 \mu \mathrm{g})$ discs were placed on the inoculated MuellerHinton agar plates. Crude enzyme was extracted from nutrient agar slope culture of the tested isolate as described previously (Livermore et al., 1984). A linear slit and a circular well were cut in the agar as described in conventional method 1 . The well was loaded with 30 to $40 \mu \mathrm{l}$ of the enzyme extract. The plate was kept upright for 5 to $10 \mathrm{~min}$ until the solution dried, and was then incubated at $37^{\circ} \mathrm{C}$ overnight (Manchanda and Singh, 2003).

Two different kinds of result were recorded in phenotypic methods. The isolates showing clear distortion of zone of inhibition of cefoxitin were taken as AmpC $\beta$-lactamase producers. The isolates with no distortion were taken as $A m p C \beta$-lactamase nonproducers.

\section{Detection of ampC genes using PCR}

Different ampC $\beta$-lactamase genes namely: AmpC, CIT-M, Fox-1, $A C C-1$ and $A C T-1$, were detected. Each was detected with gene specific primer, the sequences and the amplicon size of which are listed in Table 1. The method adapted was colony PCR. Briefly, single colony was picked up from our collected isolates and grown on LB broth medium overnight at $37^{\circ} \mathrm{C}$. Different amounts (50 to $100 \mu \mathrm{l})$ were plated on selective LB agar plates. The plates were incubated for 16 to $18 \mathrm{~h}$ at $37^{\circ} \mathrm{C}$. Subsequently, 2 to 4 colonies of each plate were randomly picked with sterile toothpicks and heated in $50 \mu \mathrm{H}_{2} \mathrm{O}$ for $5 \mathrm{~min}$ at $95^{\circ} \mathrm{C}$. Following centrifugation at 13000 rpm for $1 \mathrm{~min}$, direct colony PCR of the supernatant was performed with Dream Taq $^{\mathrm{TM}}$ Green PCR Master Mix (Fermentas). PCR cycling parameters were as follows: initial denaturation at $95^{\circ} \mathrm{C}$ for $5 \mathrm{~min} ; 40$ cycles at $94^{\circ} \mathrm{C}$ for $20 \mathrm{~s}, 60^{\circ} \mathrm{C}$ for $30 \mathrm{~s}$ and $72^{\circ} \mathrm{C}$ for $45 \mathrm{~s}$ with a final extension at $72^{\circ} \mathrm{C}$ for $7 \mathrm{~min}$. An aliquot of each reaction was analyzed on a $1.2 \%$ agarose gel (Agarose MP) stained with ethidium bromide and compared with 100 bp plus DNA Molecular Weight Marker to verify the success of the PCR reaction.

\section{Statistical analysis}

The phenotypic methods were compared using Chi square test. Statistical calculations were carried out using the Instat-2 computer program (GraphPad Software Inc. V2.04, San Diego, Calif., USA). $\mathrm{P}<0.05$ was considered to be statistically significant.

\section{RESULTS}

Detection of AmpC activity using different phenotypic methods and PCR indicates that $122(67.7 \%)$ of the tested isolates exhibited AmpC activity. Although 108 $(60 \%)$ isolates were positive by PCR, only 57 (31.6\%) were positive by different phenotypic methods. 
Table 1. Specific amplification primer sets for the tested AmpC $\beta$-lactamase genes.

\begin{tabular}{lllc}
\hline Gene name & Type & Sequence & Amplicon size (bp) \\
\hline \multirow{2}{*}{ AmpC } & Fw & ACACGAGTTTGCATCGCCTG & 254 \\
& Rv & CTGAACTTACCGCTAAACAGTGGA AT & \\
CIT-M & Fw & TGGCCAGAACTGACAGGCAAA & 462 \\
& Rv & TTTCTCCTGAACGTGGCTGGC & \\
Fox-1 & Fw & GCAAACCAGCAATACCATCCA & \multirow{2}{*}{642} \\
& Rv & GCTCACCTTGTCATCCAGCTC & \\
ACC-1 & Fw & AGCTGTTATCCGTGATTACCTGTCT & 248 \\
& $\mathrm{Rv}$ & AGCGAACCCACTTCAAATAACG & \\
& & & \\
ACT-1 & Fw & CATGCTGGATCTGGCAACCT & 343 \\
\hline
\end{tabular}

Fw: Forward, Rv: Reverse

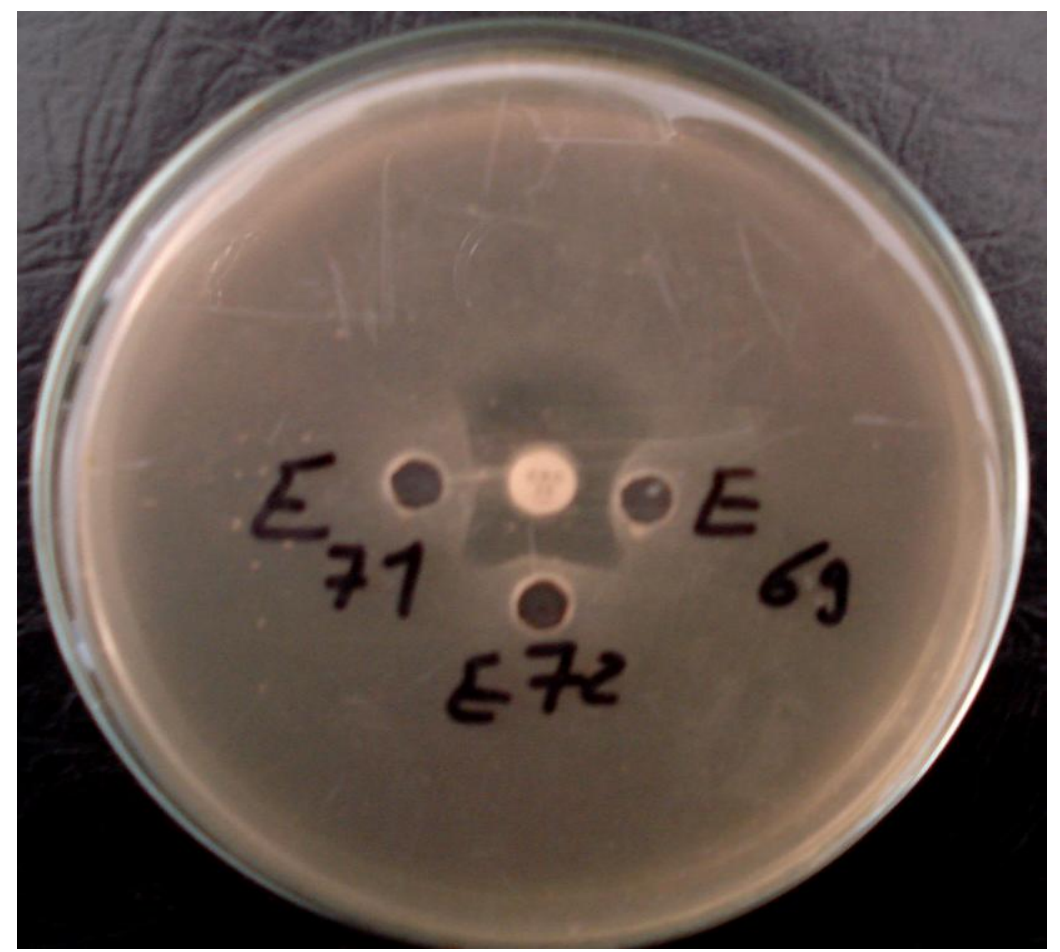

Figure 1. Detection of AmpC enzymes production using enzyme extract method. The enzyme extracts of $E$. coli isolate No. 71 and 72 were loaded in wells made at a distance of 10 to $12 \mathrm{~mm}$ from cefoxitin disc with linear slits (both enzymes exhibited distortion of inhibition zone "positive result". The enzyme extract of the E. coli isolate No. 69 was loaded in a well made at a distance of 10 to $12 \mathrm{~mm}$ from cefoxitin disc without linear slits "it shows positive result".

Regarding the phenotypic methods for detection of AmpC production, enzyme extract method showed the best results where 43 isolates exhibited clear distortion in the inhibition zone of cefoxitin and were considered posi- tive AmpC producers (Figure 1).

Similar results were shown with the method using broth suspension of the tested isolates where 39 isolates were positive AmpC producers by this method. But, only 25 of 


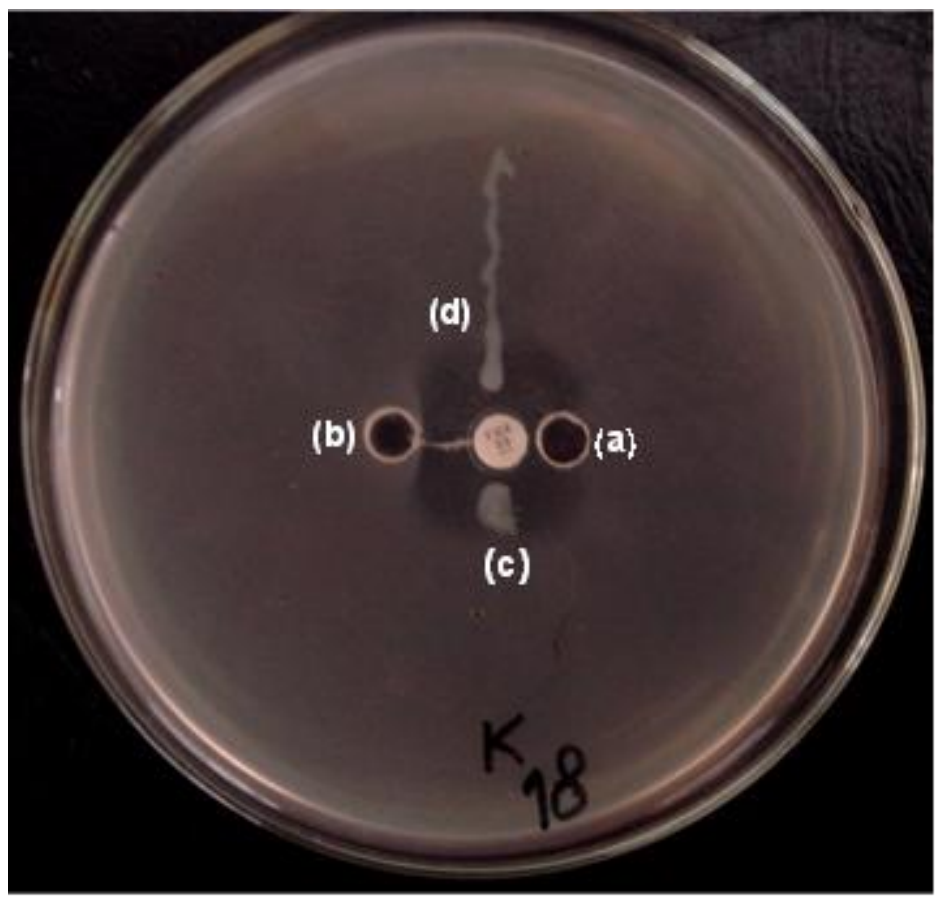

Figure 2. Detection of $A m p C$ enzymes production directly using the Klebsiella isolates (no. 18): (a) Bacterial suspension loaded in a well made within the inhibition zone without linear slit (it shows clear distortion of inhibition zone indicating positive results); (b) bacterial suspension loaded in a well made approximately at the margin of the inhibition zone with linear slit (it shows clear distortion of inhibition zone); (c) spot inoculation of the tested isolate at a distance of $6 \mathrm{~mm}$ from the edge of the centrally placed cefoxitin disc (it shows no distortion of inhibition zone); (d) streaking of the test organism over the surface of the agar (it shows no distortion of inhibition zone).

Table 2. Statistical analysis of phenotypic methods for detection of AmpC $\beta$-lactamase.

\begin{tabular}{lll}
\hline Methods & Significance \\
\hline M3D using the bacterial suspension & enzyme extract & Non statistically significant \\
Streaking culture & spot inoculation & Non statistically significant \\
Enzyme extract & streaking culture & Moderate statistically significant $(P$ value $=0.0064)$ \\
Enzyme extract & spot inoculation & High statistically significant $(P$ value $=0.0004)$ \\
M3D using the bacterial suspension & streaking culture & Low statistically significant $(P$ value $=0.0255)$ \\
M3D using the bacterial suspension & spot inoculation & Moderate statistically significant $(P$ value $=0.0024)$ \\
All phenotypic methods used for detection of AmpC. & High statistically significant $(P$ value $=0.0006)$ \\
\hline M3D: modified three dimensional & &
\end{tabular}

these isolates were considered positive by enzyme method (Figure 2).

In contrast, detection of AmpC activity using streaking culture (method 2) and spot-inoculation (method 3) showed poor results with only 23 and 18 isolates showing clear distortion of zone of inhibition of cefoxitin by the two methods, respectively (Figure 2 ).

The four phenotypic methods were statistically significant from each other as $P=0.0006$. However, upon comparing the M3D using the bacterial suspension and the enzyme extract only, it was found that they showed no significant difference. In addition, the method that used streak culture and the one that used spot inoculation were also statistically non significant from each other. Table 2 illustrates the statistical analysis of all phenotypic method.

From the distribution of different AmpC $\beta$-lactamase genes among the clinical isolates (Table 3 ), it was observed that enzymes from the AmpC group were predominantly present in $E$. coli isolates (62\%). In contrast, it was 
Table 3. Distribution of different $A m p C$ genes within the study isolates.

\begin{tabular}{lcl}
\hline Organism & Number of isolate & AmpC type \\
\hline \multirow{4}{*}{ E. coli } & 39 & AmpC \\
& 1 & AmpC, CIT-M \\
& 3 & Fox-1 \\
& 4 & AmpC, CIT-M, Fox-1 \\
& 10 & AmpC, Fox-1 \\
Klebsiella & & \\
pneumonia & 9 & Fox-1 \\
& 3 & Fox-1, ACT-1 \\
& 4 & ACC-1 \\
& 9 & ACT-1 \\
Enterobacter cloacae & 1 & Fox-1, AmpC \\
& 7 & Fox-1 \\
& 1 & ACT-1, AmpC \\
& 2 & Fox-1, ACC-1 \\
& 11 & Fox-1, ACT-1 \\
& 1 & Fox-1, ACT-1, AmpC \\
& 3 & Fox-1, ACC-1, ACT-1 \\
\hline
\end{tabular}

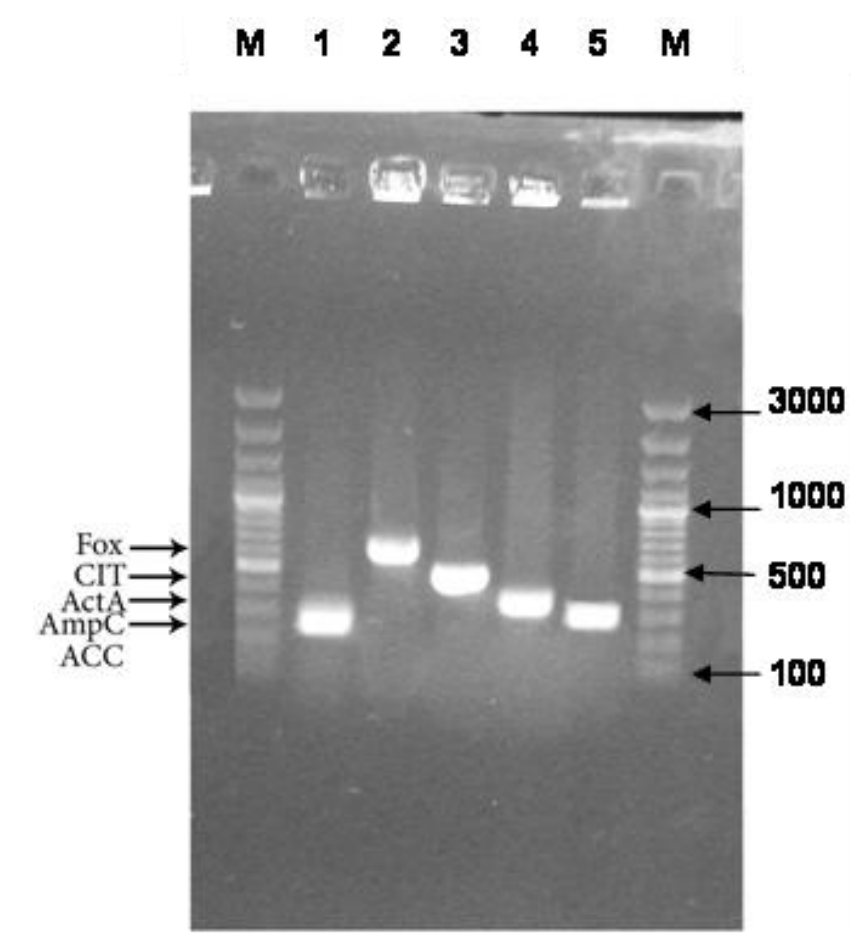

Figure 3. Agarose gel electrophoresis of five AmpC $\beta$-lactamase genes amplified from different isolates. Lane $M$ is $100 \mathrm{bp}$ DNA marker. Lanes from 1 to 5 are the amplified product of $A m p C$ (254 bp), Fox-1 (642 bp), CIT-M (462 bp), ACT-1 (343 bp) and ACC-1 (248 bp) respectively. The amplified product from each PCR is indicated on the left, and the size of the marker in base pairs is shown on the right.

present in a minority of Enterobacter cloacae isolates (9.3\%). Fox-1 like enzymes was predominantly present in
E. cloacae (89.2\%) but was also found in $E$. coli isolates $(19.5 \%)$ and Klebsiella pneumoniae isolates (19.6\%). Enzymes from $A C T-1$ predominantly present in Enterobacter cloacae $(50 \%)$ but were also found in $K$. pneumonia (19.6\%). On the other hand, it was found that enzymes from CIT-M and ACC-1 group were rarely present in Enterobacteriaceae as they were found in only 5 and 9 isolates, respectively. The amplification of products for each family member of a particular set resulted in a single amplicon of the predicted size. For example, the amplified product of the Fox-1 family resulted in large amplicon of $642 \mathrm{bp}$. In addition, single amplicons of 462 and $343 \mathrm{bp}$ were generated for the family members CIT$\mathrm{M}$ and $A C T-1$, respectively. The small amplicon of 254 and 248 were generated for the family members $A m p C$ and $A C C$ - 1 respectively (Figure 3 ).

\section{DISCUSSION}

With the spread of AmpC producing strains all over the world, it was necessary to estimate the prevalence of these strains in hospitals. Detection of resistant isolates would allow doctors to formulate a policy of empirical therapy in high-risk units where infections due to resistant organisms are much higher. Therapeutic and infection control considerations argue that recognition of plasmidmediated AmpC enzymes is necessary for the average laboratory. AmpC-producing isolates may appear to be susceptible in vitro to some cephalosporins and aztreonam yet fail to respond if those agents are used, so that a specific test for their presence is necessary (Thomson, 2001). 
In this study, we evaluated the accuracy of various phenotypic techniques used to screen for AmpC activity among Enterobacteriaceae.

Thomson and Sanders (1992) first reported threedimensional test, since then, many modifications have been made. The original method was intricate as it needed customized low-speed rotator and careful loading of slit without spilling the extract. Coudron et al. (2000) modified the original method; they used linear slits instead of circular slits. The method could overcome the need for rotator, but filling these slits without spillage was not solved. Subsequently, Manchand and Singh (2003) modified the procedure; they created a well at the outer edge of the slit and the enzyme extract was put into the well. The method overcame all the problems of threedimensional test. But the M3D test is laborious, technically demanding requiring careful cutting of slit and well, time consuming and needs experience. Streaking culture and spot inoculation test (Shahid et al., 2004) with direct bacterial colonies were introduced to overcome these problems. But these tests were less sensitive than M3D test.

To determine the placement of wells, more experiments were designed. The zone of inhibition of cefoxitin discs for the inoculated $E$. coli was noted and the wells were punched within the zone of inhibition (5 to $6 \mathrm{~mm}$ from the edge of discs), at the zone of inhibition (9 to $10 \mathrm{~mm}$ ) and outside the margin of the zone of inhibition. More positive results were obtained with wells punched within, and at the zone of inhibition.

Phenotypic methods adapted in this study gave variable results. The most discriminatory results were given by both direct inoculation of the enzyme extract in the wells made in agar and by direct inoculation of the organism in the wells. Of these, the best results were given by enzyme inoculation methods where 43 isolates exhibited positive result by this method. A major draw back of using direct inoculation of bacterial suspension is that it is not specific for AmpC activity where cefoxitin resistance may also be mediated by alterations to outer membrane permeability (Hernandez-Alle et al., 2000). This can explain the presence of 14 isolates exhibited AmpC activity by suspension method, and is considered negative by enzyme inoculation method and PCR.

All phenotypic methods used in this study detect 57 isolates as positive AmpC producer (31.6\%) but they are unable to differentiate the different types or families of AmpC $\beta$-lactamases (Thomson et al., 1991). Therefore, it is necessary to use other techniques for confirmation and detection of different AmpC gene.

We reported here the use of PCR as simple, rapid and accurate technique for confirmation of phenotypic methods and discrimination of different AmpC gene among different isolates. Although 57 isolates were reported as positive AmpC producer by phenotypic methods, detection by PCR indicated that 108 isolates were positive $A m p C$ producer. This great variation in the results ob- tained by phenotypic detection and by PCR may be due to the fact that chromosomal AmpC genes present in $E$. coli, are not usually expressed because of the presence of a transcriptional attenuator coupled with a weak promoter (Tracz et al., 2007). Therefore, not all isolates detected as AmpC producers by PCR can be detected by different phenotypic methods.

In conclusion, this report evaluated several phenotypic methods for screening of AmpC enzymes and demonstrated that using enzyme inoculation method accurately detects the AmpC-positive isolates. For confirmation of AmpC-positive isolates and discrimination of different AmpC genes among different isolates, PCR can be used as simple, rapid and accurate technique.

Further studies will be needed to establish the reliability of these methods in detecting AmpC enzymes among other pathogens, such as Pseudomonas aeruginosa, as well as among pathogens possibly coproducing extended-spectrum $\beta$-lactamases.

\section{ACKNOWLEDGEMENT}

Authors wish to express gratitude and deep thanks to Dr. Ghada Sidek at Pharmacology department, Faculty of Pharmacy, Mansoura University, Mansoura, Egypt for her valuable cooperation to perform the statistical analysis.

\section{REFERENCES}

Barlow M, Hall BG (2002). Origin and evolution of the AmpC $\beta$ lactamases of CIT-M Citrobacter freundii. Antimicrob. Agents Chemother. 46: 1190-1198.

Chen YT, Lauderdale TL, Liao TL, Shiau YR, Shu HY, Wu KM, Yan JJ, Su IJ, Tsai SF (2007). Sequencing and comparative genomic analysis of pK29, a 269-kilobase conjugative plasmid encoding CMY8 and CTX-M-3 $\beta$-lactamases in Klebsiella pneumoniae. Antimicrob. Agents Chemother. 51: 3004-3007.

Coudron PE, Hanson ND, Climo MW (2003). Occurrence of extended spectrum and AmpC $\beta$ - lactamases in blood stream isolates of Klebsiella pneumoniae: isolates harbor plasmid -mediated Fox-5 and ACT-1 AmpC $\beta$-lactamases. J. Clin. Microbiol. 41: 772-777.

Coudron PE, Moland ES, Thomson KS (2000). Occurrence and detection of AmpC $\beta$-lactamases among Escherichia coli, Klebsiella pneumoniae and Proteus mirabilis isolates at a Veterans Medical Center. J. Clin. Microbiol. 38: 1791-1796.

Crichton PB (1996). Enterobacteriaceae: Escherichia, Klebsiella, Proteus and other genera. In Mackie and Mccartney Practical Medical Microbiology, $14^{\text {th }}$ edn. (Collee Jg, Fraser AG, Marmion BP, Simmons A, Eds), PP. 361- 384. Churchill living-stone- EbionburghU.K.

Hernandez-Alles S, Conejo M, Pascual A, Tomas JM, Benedi VJ, Martinez-Martinez L (2000). Relationship between outer membrane alterations and susceptibility to antimicrobial agents in isogenic strains of Klebsiella pneumoniae. J. Antimicrob. Chemother. 46: 273277.

Jacoby GA (2009). AmpC $\beta$-lactamase. Clinical Microbiology Reviews. 22(1):161- 182.

Livermore DM, Maskell JP, Williams JD (1984): Detection of PSE-2 $\beta$ lactamase in enterobacteria Antimicrob. Agents Chemother. 25: 268272.

Manchanda V, Singh NP (2003). Occurrence and detection of AmpC $\beta$ lactamases among Gram-negative clinical isolates using a modified three-dimensional test at Guru Tegh Bahadur Hospital, Delhi, India. J. Antimicrob. Chemother. 51: 415-418. 
Odeh R, Kelkar S, Hujer AM, Bonomo RA, Schreckenberger PC, Quinn $J P(2002)$. Broad resistance due to plasmid-mediated AmpC $\beta$ lactamases in clinical isolates of Escherichia coli. Clin. Infect. Dis. 35: 140-145.

Shahid M, Malik A, Agrawal M, Singhal S (2004): Phenotypic detection of extended-spectrum and AmpC $\beta$-lactamases by a new spotinoculation method and modified three-dimensional extract test: comparison with the conventional three-dimensional extract test. J. Antimicrob. Chemother. 54: 648-687.

Thomson KS (2001). Controversies about extended-spectrum and AmpC $\beta$ - lactamases. Emerg. Infect. Dis. 7: 333-336.

Thomson KS, Sanders CC (1992): Detection of Extended-spectrum $\beta$ lactamases in members of the family Enterobacteriaceae: Comparison of double-disk and three-dimensional tests. Antimicrob. Agents Chemother. 36:1877-1882.
Thomson KS, Sanders CC, Washington JA II (1991). High-level resistance to cefotaxime and ceftazidime in Klebsiella pneumoniae isolates from Cleveland, Ohio. Antimicrob. Agents Chemother. 35: 1001-1003.

Tracz DM, Boyd DA, Hizon R, Bryce E, McGeer A, Ofner-Agostini M, Simor AE, Paton S, Mulvey MR (2007). AmpC gene expression in promoter mutants of cefoxitin-resistant Escherichia coli clinical isolates. FEMS Microbiol. Lett. 270: 265-271. 